\title{
A Randomized Controlled Clinical Study of Irinotecan/ cisplatin Regimen and Etoposide/cisplatin Regimen in the First-line Treatment of Small Cell Lung Cancer
}

Bo Jiang

Sihong Geriatrics Hospital, Sihong 223900, Jiangsu Province, China

Abstract: Objective: This paper mainly study on the effects of irinotecan/cisplatin and etoposide/ cisplatin regimens in the treatment of small cell lung cancer. Methods: 50 cases of small cell lung cancer patients in our hospital were divided into control group and experimental group and administered with etoposide/cisplatin and irinotecan/cisplatin regimen, and the treatment effects of the two regimens were compared statistically. Results: After treatment, both groups achieved high treatment efficiency, and the incidence of toxic side effects was low, with no significant difference $(P>0.05)$; serological ABCG2 and FGFBP1 level indicators were better than the control group, both showing significant differences $(P<0.05)$. Conclusions: Irinotecan has achieved better improvement in serological indicators in the first-line treatment of small cell lung cancer, with no significant difference in short-term treatment efficiency.

Keywords: Irinotecan; Cisplatin; Etoposide; Small cell lung cancer

Publication date: November, 2020

Publication online: 30 November, 2020

"Corresponding author: Bo Jiang, 798582038@ qq.com

Lung cancer is a malignant tumor disease with a high mortality rate in the world and small cell lung cancer is one of the commonly seen clinical disease. Small cell lung cancer has a relatively low degree of pathological differentiation and a great capability to infiltrate and metastasize, so distant metastasis would have already occurred when the disease is diagnosed in most of its patients, causing tremendous harm to patients' health ${ }^{[1-2]}$. Currently, chemotherapy is a major treatment method for small cell lung cancer. Previously, combinations of cisplatin with related drugs were often used for treatment. Among them, etoposide and irinotecan were more commonly used. Both drugs when combined with cisplatin can achieve certain treatment effects. Based on this, this paper selects the treatment effects of irinotecan/cisplatin and etoposide/cisplatin for small cell lung cancer patients in our hospital for comparative analysis to summarize relevant experience.

\section{Background and Methodology}

\subsection{Basic Background}

Since February 2016 till March 2020, our hospital's medical staff carried out a 4-year study with a total number of 50 small cell lung cancer patients being accepted as research subjects during this period. All research subjects have been ruled out of cardiovascular and cerebrovascular diseases and severe immune dysfunction, and all of them have voluntarily signed the research consent form, which has been reviewed and approved by the ethics committee. The patients were divided into the control group and the experimental group by drawing lots, each with 25 cases. The male to female ratio of the control group was $14: 11$, and the age range was 57 78 , with average age being $(65.13 \pm 1.42)$ years old; the male to female ratio of the experimental group was 13:12, and the age range was 56-77, with average age being $(65.22 \pm 1.39)$ years old; statistical analysis shows no significant differences 
between the two groups $(P>0.05)$.

\subsection{Methods}

The control group was treated with etoposide/ cisplatin regimen. Firstly, etoposide $100 \mathrm{mg} /\left(\mathrm{m}^{2} \bullet \mathrm{d}\right)$ provided by Qilu Pharmaceutical Co. Ltd. was mixed with normal saline for intravenous infusion, and the infusion time was controlled at 30 minutes and above. Simultaneously, cisplatin $25 \mathrm{mg} /\left(\mathrm{m}^{2} \bullet \mathrm{d}\right)$ provided by Qilu Pharmaceutical Co. Ltd. was administered through intravenous dripping on the 13th day with hydration and dieresis given before administration. 2 courses of treatment were given and each course lasted 21 days ${ }^{[3]}$.

The experimental group was treated with the iritecan/cisplatin regimen. Firstly, iritecan $60 \mathrm{mg} /$ $\left(\mathrm{m}^{2} \bullet \mathrm{d}\right)$ provided by Qilu Pharmaceutical Co. Ltd. was administered through intravenous dripping and cisplatin $25 \mathrm{mg} /\left(\mathrm{m}^{2} \bullet \mathrm{d}\right)$ was given on $1 \mathrm{st}$ and 8 th day. 2 courses of treatment were given and each course lasted 21 days.

\subsection{Observations of the Indicators}

The levels of serological ABCG2 and FGFBP1 before and after treatment in the two groups were observed statistically; the short-term treatment efficiency of the patients was evaluated using the WHO response evaluation criteria in solid tumors (RECIST), which mainly included four levels, namely complete remission, partial remission, stable and progress. The total efficiency $=($ Complete remission + partial remission)/total number of cases $\times 100 \%$; at the same time the incidence of side-effects such as nausea and vomiting, diarrhea, leukopenia and thrombocytopenia were observed.

\subsection{Statistical Analysis}

Data was calculated using SPSS23.0, with $\mathrm{t}$ and X2 tests performed and presented in $(\bar{x} \pm s) \quad(n / \%)$, if $P<0.05$, it means that there is difference in data.

\section{Results}

\subsection{Statistical Assessment of Serological Indicators Before and After Treatment}

It is obvious from the data in Table 1 that there was no significant difference between the two groups before treatment. After treatment, the levels of ABCG2 and FGFBP1 of the experimental group were both significantly lower than those of the control group, with $P<0.05$.

Table 1. Statistical Assessment of ABCG2 and FGFBP1 Indicators $(\bar{x} \pm s)$

\begin{tabular}{ccccccc}
\hline \multirow{2}{*}{ Group } & \multirow{2}{*}{$\boldsymbol{n}$ (sample size) } & \multicolumn{2}{c}{ ABCG2 } & & \multicolumn{2}{c}{ FGFBP1 } \\
\cline { 3 - 4 } \cline { 5 - 7 } & & Before treatment & After treatment & & Before treatment & After treatment \\
\hline Control Group & 55 & $223.13 \pm 9.84$ & $208.43 \pm 8.47$ & & $42.86 \pm 5.47$ & $25.44 \pm 3.81$ \\
Experimental Group & 55 & $224.05 \pm 9.71$ & $165.68 \pm 8.51$ & & $42.97 \pm 5.34$ & $16.18 \pm 3.15$ \\
$T$ & & 0.4935 & 26.4054 & 0.1067 & 13.8916 \\
$P$ & & 0.6226 & 0.0000 & 0.9152 & 0.0000 \\
\hline
\end{tabular}

2.2 Statistical Assessment of Treatment Efficiency in the Two Groups

Statistical analysis shows that both groups of patients achieved higher treatment efficiency with $P>0.05$.

Table 2. Statistical Assessment of Treatment Efficiency in the Two Groups ( $n / \%)$

\begin{tabular}{ccccccc}
\hline Group & $\boldsymbol{n}$ (sample size) & Complete Remission & Partial Remission & Stable & Progress & Total Efficiency \\
\hline Control Group & 25 & 6 & 10 & 6 & 3 & 64.00 \\
Experimental Group & 25 & 7 & 11 & 5 & 2 & 72.00 \\
$\chi^{2}$ & & & & & 0.3676 \\
$P$ & & & & 0.5442 \\
\hline
\end{tabular}

2.3 Statistical Assessment on the Incidence of Sideeffects in the Two Groups

After statistical analysis, it can be seen that the incidence of various toxic side effects in both the experimental group and the control group is low and there is no significant difference between the two $(P>0.05)$. 
Table 3. Statistical Observation of the Incidence of Side-effects

\begin{tabular}{ccccc}
\hline Group & $n$ (sample size) & Nausea & Neutropenia & Vomit \\
\hline Control Group & 25 & $3(12.00)$ & $2(8.00)$ & $1(4.00)$ \\
Experimental Group & 25 & $2(8.00)$ & $0(0.00)$ & $0(0.000)$ \\
X2 & & 0.2222 & 2.0833 & 1.0204 \\
P & & 0.6373 & 0.1489 & 0.3124 \\
\hline
\end{tabular}

\section{Discussions}

Small cell lung cancer represents about 15\%-20\% of all lung cancer diseases. The disease has a high degree of malignancy and the tumor grows faster. It easily invades the lymphatic and blood capillaries of patients in the early stage, leading to systemic dissemination. Currently, it is generally believed that etoposide/cisplatin is still an effective method for the treatment of small cell lung cancer in clinical practice. Irinotecan (CPT-11) is a new type of semi-synthetic soluble camptothecin derivatives that can affect the S-phase of the cell cycle and inhibits corresponding enzymes, thereby having a positive impact on the replication of DNA molecules. It is a substance with unique molecular structure and has achieved specific therapeutic effects in clinical practice. High concentrations of irinotecan can effectively disrupt DNA transcription causing the death of cells in other phases. The application of irinotecan in the treatment of cervical cancer, colon cancer and other cancers has achieved remarkable results ${ }^{[4-5]}$. According to relevant studies, irinotecan mainly produces diarrhea and other side effects. There are two highincidence periods of diarrhea induced by the drug and the active metabolite SN-38. Usually, early diarrhea will occur in patients within 1 day after drug infusion and it is believed that it may be related to the excitation of cholinergic nerves caused by the druginduced inhibition of cholinesterase activity. It may be accompanied by sweating, flushing, tearing and excessive salivation, but these adverse reactions are mostly transient. Occasionally the reactions may be more severe and reasonable prevention and treatment can be achieved by giving atropine and other drugs to the patients. The other type of diarrhea is the delayed diarrhea, which mainly occurs on the fifth day after drug administration and it will last for about 4 days. It is generally believed that it is caused by the excessive secretion of water electrolyte in the intestinal cavity due to abnormal ion transport induced by damage of intestinal mucosa.

Clinically, the problem of diarrhea can be effecti- vely improved after oral administration of loperamide and other drugs. As a heavy metal complex, cisplatin can effectively inhibit deoxyribonucleic acid and exert certain inhibitory effects on high-concentration protein, sputum and cough, so it is often used in conjunction with other drugs.

In recent years, FGFBP1 has been a focus for tumor cells studies. It was first discovered after being isolated from epidermal squamous cell carcinoma culture medium. It is highly related to the fibroblast growth factor family and it gradually increases in lung cancer tissues, promoting the continuous rapid proliferation of fibrous tissue and benefitting the growth of tumor cells ${ }^{[6-8]}$. According to related research, increased FGFBP1 can be beneficial to tumor cell growth, thereby making new blood vessels more likely to appear, providing more adequate blood supply to tumor tissues and ultimately promoting tumor cell growth and metastasis. It can be seen from the result of this study that after treatment, both the control group and the experimental group achieved better therapeutic effects, and the toxicity was lower, with no significant difference between the two groups $(P>0.05)$; the experimental group's serum FGFBP1 and ABCG2 indicator levels were lower than the control group and the difference between the two groups is significant $(P<0.05)$.

In conclusion, irinotecan has achieved better improvement in serological indicators in the first-line treatment of small cell lung cancer, with no significant difference in short-term treatment efficiency.

\section{References}

[1] Chen Li, Yang H, Xiao GY, et al. Clinical Study on Treatment of Small Cell Lung Cancer with Injection of Irinotecan/ cisplatin[J]. Journal of Hebei North University (Natural Science Edition), 2019, 35(6): 12-14,16.

[2] Liang C, Chang HZ, Lei P et al. The effect of irinotecan on the expression of FGFBP1 and ABCG2 in serum in the treatment of small cell lung cancer[J]. International Journal of Respiration, 2019, 39(23): 1766-1768.

[3] Lu H, Kang MH, Yi L, et al. Efficacy of irinotecan/cisplatin and etoposide/cisplatin combined with concurrent thoracic 
radiotherapy in patients with extensive stage small cell lung cancer [J]. Guizhou Medical Journal, 2018, 42(7): 835-836.

[4] Hong Zhou, Shun Li Ji, Jian Hua Xu, et al. Effect of cisplatin combined with irinotecan and etoposide on lung function of patients with small cell lung cancer and its therapeutic effect [J].Anti-tumor Pharmacy,2018,008(006):885-888.

[5] Mi Wang, Hai Bo Li. A retrospective analysis of the efficacy and side effects of irinotecan combined with nedaplatin compared with cisplatin in the second-line treatment of relapsed or refractory small cell lung cancer [J].Systems Medicine, 2018,003(022):P.34-35.

[6] Ai QM, Bao QL, Ai JS et al. Study on the safety and effectiveness of various second-line chemotherapy regimens in the treatment of sensitive reccurent small cell lung cancer $[\mathrm{J}]$. Oncology Progress, 2018, 16(7): 850-853.

[7] En JX, Guo JL. Clinical study of Kang-ai injection combined with irinotecan and carboplatin in the treatment of extensivestage small cell lung cancer[J]. Drugs and Clinic, 2018, 33(10): 188-191.

[8] Chen L, Xiao GY, Yang H, et al. Effect of irinotecan combined with carboplatinum on the serum levels of patients with extensive-stage small cell lung cancer [J]. Anti-tumor Pharmacy, 2018, 8(2): 211-214. 